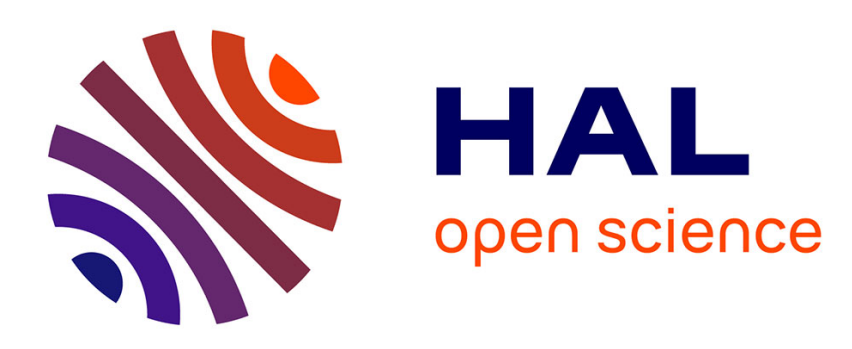

\title{
NATURE, FONCTION ET INDISPENSABILITE DES ASSOMPTIONS CHEZ MEINONG
}

\author{
Bruno Langlet
}

\section{To cite this version:}

Bruno Langlet. NATURE, FONCTION ET INDISPENSABILITE DES ASSOMPTIONS CHEZ MEINONG. Philosophie, 2019, 142, pp.30-53. hal-02397026

\section{HAL Id: hal-02397026 \\ https://hal-amu.archives-ouvertes.fr/hal-02397026}

Submitted on 6 Dec 2019

HAL is a multi-disciplinary open access archive for the deposit and dissemination of scientific research documents, whether they are published or not. The documents may come from teaching and research institutions in France or abroad, or from public or private research centers.
L'archive ouverte pluridisciplinaire HAL, est destinée au dépôt et à la diffusion de documents scientifiques de niveau recherche, publiés ou non, émanant des établissements d'enseignement et de recherche français ou étrangers, des laboratoires publics ou privés. 


\section{BRUNO LANGLET \\ NATURE, FONGTION ET INDISPENSABILITE DES ASSOMPTIONS GHEZ MEINONG}

(Pénultième version d'un article paru dans la revue Philosophie, éditions de Minuit, $\mathrm{n}^{\circ} 142$, Juin 2019, p. 30-53)

\section{INTRODUCTION}

Les attitudes psychiques, considérées sous leur aspect épistémique, consistent dans certains actes mentaux et expériences d'un sujet orienté vers quelque chose du monde et déterminent des processus conduisant à des situations de cognition et d'action. Un enjeu, ici, est de déterminer quelle liste d'attitudes est à la fois économe et pertinente. Quelques attitudes principales sont généralement reconnues : ainsi les croyances, suppositions, représentations, jugements, émotions, désirs. Celles-ci, classiques, peuvent pourtant être ramenées à quelques-unes qui permettraient de les penser. Brentano s'en tenait par exemple aux représentations, jugements et sentiments. Si cette tripartition a eu une influence remarquable sur le développement de la philosophie autrichienne, ainsi que sur celui de ses ramifications analytiques et phénoménologiques, elle doit malgré tout être interrogée : permet-elle de rendre compte des implications psychiques, épistémologiques et ontologiques qui se trouvent être en jeu lors des processus ordinaires de pensée ? C'est ce qui a été contesté, au début du $\mathrm{XX}^{\mathrm{e}}$ siècle, par le philosophe Alexius Meinong, qui a plaidé en faveur de l'ajout de la notion d'Annahmel (assomption) à la liste des attitudes psychiques fondamentales. Dans sa perspective, les assomptions sont essentielles à la description et à la compréhension des processus menant à la connaissance, mais aussi de ceux par lesquels des objets de pensée peuvent être visés et saisis par l'esprit, antérieurement à la question de savoir s'ils existent ou s'ils peuvent être correctement pensés sur un mode véritatif. Par cette notion d'assomption, il faut entendre une attitude psychique tournée vers certains objets de pensée qui, par là, sont «admis » afin d'être considérés par l'esprit, sans que cette « admission » n’implique un engagement envers leur existence. Le concept d'assomption a ainsi pour Meinong un légitime droit de cité en

\footnotetext{
${ }^{1}$ (Merci à Pascal Engel et à Jean-Maurice Monnoyer.) En 1902, Meinong fait paraître son important texte Über Annahmen, puis une deuxième édition en 1910, considérablement remaniée. Über Annahmen, 1902, 1910², Gesamtausgabe, Bd. IV, éd. Haller \& Kindinger, 1968. Pour une présentation classique de l'oeuvre de Meinong, voir John Niemeyer FINDLAY, Meinong's Theory of Objects and Values, Oxford, Clarendon Press, $1963^{2}$; Reinhardt Grosmann, Meinong, The arguments of the philosophers, London-Boston, Routledge and Kegan Paul, 1974.
} 
psychologie et en philosophie, car il est indispensable pour expliquer l'activité de l'esprit envers certains types d'objets et certaines modalités de l'existence ou de la non-existence dont ils sont solidaires ${ }^{2}$. Faute de le reconnaître, on court ainsi le risque d'utiliser incorrectement les notions de jugements, de représentations ou de sentiments lorsqu'il s'agit de rendre compte de certaines activités de l'esprit envers certains types d'objets pour lesquels seule la notion d'assomptions serait appropriée.

Le statut de ces assomptions n'est toutefois pas immédiatement clair. Ge sont des attitudes propositionnelles qui n'impliquent pas la conviction épistémique que l'on peut trouver dans les jugements ou les croyances, bien qu'elles portent sur des états de choses (ou complexes structurés) alors présentés à l'esprit, et quoique cette visée puisse être positive ou négative. Ces attitudes ne possèdent pas non plus ce que l'on pourrait penser essentiel à toute activité psychique à visée épistémique, c'est-à-dire cette forme de pénétration sérieuse et caractéristique de la tentative d'appréhender le réel, précisément parce qu'elles sont fondamentalement tournées vers ce qui n'est pas. Il pourrait être ici avancé qu'elles ne sont que des formes de conjectures ou de suppositions. Meinong signale toutefois que ces dernières ont des conditions de correction: elles en viennent, à un moment ou un autre, à être confrontées au réel, tandis que ce n'est pas le cas des assomptions qui sont cognitivement destinées à autre chose. Par exemple, me promenant le long du Danube, je peux supposer qu'il ne va pas y avoir de crue à la suite d'indices météorologiques et de ce que je vois des aménagements réalisés par les pouvoirs publics. Ces suppositions ou conjectures à propos du Danube peuvent être falsifiées (apparition d'une crue) ou vérifiées (absence de crue). Or former l'assomption que le Danube est en crue consiste en tout autre chose : il s'agit de concevoir ici une situation fictive, ce qui revient à se donner un objet de pensée - le Danube qui sort de son lit - tout en admettant qu'il ne s'agit pas d'une situation réelle, et que cette pensée n'a par principe pas de conditions de correction. (La parenté avec l'imagination est claire, mais les assomptions peuvent aussi être très abstraites.) Il semble alors légitime de s'interroger sur le rôle de telles attitudes dans l'économie de la cognition, tout comme sur leur nature d'attitudes psychiques et épistémiques supposées rendre compte de faits épistémologiques. De manière connexe, il convient d'établir quels problèmes elles permettent de poser - ou de résoudre - et que les autres actes psychiques ne peuvent pas traiter. C'est donc aussi poser

${ }^{2}$ Comme on le voit, cette théorie des assomptions est essentielle à la compréhension de la perspective de Meinong sur les objets non existants, même si elle n'épuise pas les aspects problématiques, épistémologiques et ontologiques impliqués dans sa pensée sur ce sujet. Pour une approche explicative de ces problems, voir par exemple Peter SimOns, "On what there isn't: the Meinong-Russell dispute", in P. Simons, Philosophy and logic in Central Europe from Bolzano to Tarski : selected essays. Dordrecht, Kluwer Academic Publishers, 1992, p. 159-191 ; ou Janet Farrell SMITH, "The Russell-Meinong Debate", Philosophy and Phenomenological Research 45, 1985, pp. 305-350. 
la question de leur dispensabilité. ${ }^{3}$ Car ces interrogations naissent en raison des caractéristiques mêmes des assomptions :il est a priori difficile de les tenir pour des attitudes intrinsèquement épistémiques, car elles portent sur des objets qui sont considérés comme neutralisés ontologiquement. C'est un des points que nous discuterons en considérant des faits de connaissance qui impliquent que des assomptions soient à l'oeuvre. Par ailleurs, celles-ci ne peuvent pas ne pas entretenir certaines relations avec d'autres attitudes plus directement identifiables comme épistémiques, telles que les représentations, des jugements, les sentiments. Un certain nombre de leurs fonctions ${ }^{4}$ nous permettra de montrer en quoi elles ne se résument pas à la simple addition d'un phénomène psychique à la tripartition que Brentano avait établie. En effet, les assomptions $1 /$ permettent de rendre compte d'activités étrangères aux représentations et aux jugements, et donc, en ce sens, elles sont bien nécessaires, 2/ elles jouent aussi un rôle dans la formation des représentations et des jugements, et de ce point de vue elles ne sont pas dispensables, car elles déterminent en partie l'efficacité cognitive de ces représentations et jugements, 3/ leur fonction se manifeste de plus dans la classe des phénomènes affectifs, au sens où des « sentiments assomptifs » permettent de rendre compte, chez le Meinong de 1910, de la relation entre valeur, sentiment et désir, et de distinguer des niveaux d'évaluations.

\section{ASSOMPTIONS ET GONNAISSANCE (I) : GONSIDERER SANS (OU AVANT DE) JUGER}

Meinong n'est pas le seul à emprunter la voie des assomptions : Frege le fait aussi. En effet, chez ce dernier ${ }^{5}$, il est nécessaire de pouvoir « considérer » une proposition de manière indépendante de l'acte de juger, et donc de la viser par le moyen d'une Annahme: sans cela, nous aboutirions à l'impossibilité de penser à une proposition sans la juger ipso facto. Or Frege veut ménager la possibilité de considérer une proposition avant de décider s'il faut lui attribuer une valeur de vérité, donc avant de l'affirmer ou de la nier. C'est par

${ }^{3}$ Cette question a été posée de manière urgente et intense par les adversaires de Meinong, dès la parution de la première édition de Über Annahmen. Marty ou Brentano avaient ainsi une position claire: les assomptions sont inutiles, conduisent à des contradictions, et comme on le dirait aujourd'hui, font proliférer les entités ou objets au sein d'une certaine ontologie. Nous revenons sur cela infra.

${ }^{4}$ Nous laisserons de côté certaines dimensions qui nous semblent mieux connues: elles s'opposent aux représentations, car il n'y a pas de représentations du négatif, mais des assomptions; elles permettent d'affaiblir le rapport entre la nécessité des objectifs et la théorie de la production des idées ; elles connectent les contenus de pensée.

${ }^{5}$ Dans „Funktion und Begriff“ : « Cette séparation du jugement et de ce sur quoi l’on juge semble inévitable ; sinon on ne pourrait exprimer une simple assomption [Annahme], la position d'un cas, sans en même temps juger de son occurrence. Nous avons donc besoin d'un signe particulier pour l'affirmation. J'emploie à cette fin un trait vertical placé à gauche de l'horizontal... » (trad. fr. C. Imbert modifiée, « Fonction et concept », in Écrits logiques et philosophiques, Paris, Seuil, 1971, p. 94). 
conséquent une fonction pré-épistémique de l'assomption qui permet de constituer une zone objectuelle préalable à l'acte du jugement. Frege ne fait pas appel ici aux seules représentations pour assurer cette fonction de la considération des pensées. Tourner son attention vers une proposition impliquerait donc bien quelque chose de plus que d'en faire seulement l'objet de représentations et ne se réduirait pas à une combinaison de celles-ci. Il pourrait être ici utile de mettre ce point en question et de demander si cette zone d'articulation de pensées, préalable au jugement, n'est pas réductible, pace Frege et Meinong, à une activité consistant à construire des idées uniquement par un jeu de représentations.

Si c'est le cas, une approche de type cartésien pourrait ici être substituée à la pensée par assomptions. Descartes oppose justement processus de pensée et actes de jugement ${ }^{6}$ lorsqu'il traite des conceptions de l'entendement, tandis que le jugement est suspendu, résistant aux séductions et risques de la précipitation, et comme en attente que la plus grande clarté devienne une propriété des idées ainsi conçues. Il semble toutefois douteux que les seules représentations puissent remplir cette tâche de conception, et qu'à elles seules, elles puissent rendre compte du travail de l'entendement, lequel semble bien présupposer ce que Meinong appellera des assomptions. La pensée qui vise une idée, sans rien affirmer de sa vérité ou de sa fausseté par un jugement, mais en lui attribuant des caractéristiques, ou des relations, par exemple en tentant de concevoir l'objet de cette idée indépendamment de quelque substrat, ou en pensant à celui dont il dépend - donc en la concevant indépendamment de quelque chose ou dans sa dépendance avec ce quelque chose - n'a pas affaire ici aux seules représentations. Car celles-ci porteraient sur des objets se tenant dans certaines relations d'incompatibilité, d'inhérence, de différence, d'indépendance, de ressemblance, de causalité, autrement dit, ces représentations porteraient sur des objets qui se trouvent au sein d'états de choses.

Cela implique donc ce que nous appelons une attitude propositionnelle : considérer par exemple qu'un cristal de glace a une structure déterminée par la symétrie hexagonale, sans encore juger que c'est le cas, est quelque chose de différent de penser à la glace et à l'hexagonalité. C'est aussi différent de se représenter un cristal de glace. Dans ce contexte, un objet qui n'existe pas nécessairement, sauf en tant qu'objet de pensée, se trouve visé par l'esprit : il est affirmé par là qu'il possède une texture glacée, et qu'il y a une relation entre cette texture et la structure déterminée par la symétrie hexagonale. La pensée n'est pas ici tournée vers un morceau de glace, vers l'hexagonalité ou la notion de structure, mais vers un certain état de choses, une entité complexe dotée d'une certaine unité. De telles conceptions vont donc au delà d'un jeu de représentations visant chacune un objet

6Opéré par la volonté, dans la quatrième des Méditations métaphysiques, « Du vrai et du faux », CEuvres complètes, AT IX, p. 42-50. 
spécifique; elles semblent impliquer ce que l'on peut donc appeler avec Meinong des assomptions. La proposition ou l'objectif « le cristal de glace a une structure déterminée par la symétrie hexagonale » est une pensée ayant une unité, laquelle ne peut pas être obtenue seulement par la concaténation des représentations de la glace, de la notion de structure, de celles de la symétrie et de l'hexagonalité. Les assomptions permettent de considérer des objets de pensée et de les articuler dans des complexes, au moyen de processus ici préalables aux affirmations qui relèvent du jugement. Russell affirmait dans le manuscrit de 1913 que la distinction entre l'assomption et le jugement recouvre celle qu'il y a entre comprendre une proposition et la juger ${ }^{7}$ : comprend-on une proposition simplement en passant par des représentations? Il semble que ce ne soit pas le cas. Les assomptions ont donc bien une fonction épistémique au sens où elles font partie du processus menant à des connaissances ou à des erreurs.

Cette classe d'acte psychique, en vertu de ses caractéristiques, est aussi caractérisable comme la condition psychologique dédiée à l'usage épistémique de certains types de fictions. Les assomptions permettent de penser des états de choses sans affirmer leur existence, mais cela ne signifie pas que l'on ne puisse pas considérer des propriétés ou des relations de ces objets, de manière à obtenir un gain épistémique. Le paradoxe apparent est certes que nous nous trouvons alors dans une situation où nous voulons connaître des traits du réel, ou bien établir des raisonnements permettant de trancher des problèmes relatifs au réel, et que pour cela, nous en passons par des objets tenus d'emblée pour distincts de ce qui existe, ou par des raisonnements qui ont une forte dimension contrefactuelle. Mais il y a des candidats désormais classiques pour ces types de rôles : les expériences de pensée et les modèles paraissent les plus adéquats, à titre de fictions épistémiques, pour éclairer cette perspective, et, donc, une autre fonction des assomptions.

\section{ASSOMPTIONS ET GONNAISSANCE (II) : EXPERIENCES DE PENSEE}

Un certain nombre d'expériences de pensée sont assez célèbres en philosophie ou en science. ${ }^{8}$ Considérons l'argument de la connaissance présenté par F. Jackson dans son «Epiphenomenal qualia ${ }^{9}$ » et, plutôt que de nous intéresser à Mary dans sa chambre

\footnotetext{
${ }^{7}$ Bertrand Russell, Le manuscrit de 1913, Paris, Vrin, 2002 pour la trad. fr., Partie II, Chap. 1, p. 140-142. ${ }^{8}$ Pensons par exemple aux cas relatifs au Trolley bus de P. Foot, au linguiste de Quine s'interrogeant sur le sens du terme «Gavagai », à Mach et son cosmonaute, Avicenne et l'homme volant, au problème de Molyneux, à Locke et à l'inversion de l'identité personnelle, à Parfit et aux nombreux cas qu'il présente relatifs aussi à l'identité personnelle, à Pascal et au savetier rêvant la vie d'un roi (et inversement) pendant $12 \mathrm{~h}$, à la Terre jumelle de Putnam, au voile d'ignorance de Rawls, aux différents états de nature des philosophes classiques, aux zombies en philosophie... - liste non exhaustive.

${ }^{9}$ The Philosophical Quarterly, vol. 32, n 127 (Apr. 1982), p. 127-136.
} 
dépourvue de couleurs, prenons le cas du personnage fictif Harpo imaginé par $\mathrm{H}$. Robinson ${ }^{10}$. Harpo est frappé de surdité congénitale et passionné par la musique. Il dispose de grandes capacités intellectuelles et finit par maîtriser tout le savoir disponible sur les sons et la musique, savoir qui est ici exprimé en termes d'information physique, tel que le présentent l'ensemble des sciences. Ce savoir est une image de ce que serait notre rapport au monde s'il ne consistait qu'en propositions du physicalisme réductionniste, censé rendre compte de toute connaissance, sans omission aucune, en termes d'information physique. Cette situation fictive consiste dans un ensemble d'états de choses qui font l'objet de plusieurs assomptions enchâssées et formulées de manière précise. Il est alors possible de s'y interroger sur les conséquences épistémologiques qui suivent de l'apparition d'un nouveau fait au sein de la fiction, comme lorsque Harpo retrouve soudain l'ouie, disons par un miracle (nouvelle assomption). Il fait alors l'expérience consciente de sensations auditives, entretient une nouvelle relation en première personne avec la musique, et semble ainsi obtenir un savoir qu'il ne possédait préalablement pas et qui échappe donc à l'image physicaliste du monde. Ce type de fait épistémique conçu à l'intérieur d'une situation fictive permet classiquement de soutenir que le physicalisme ne peut pas rendre compte de toutes les connaissances, contrairement à ses prétentions de base, et qu'il est donc incomplet voire faux selon Jackson. Cet argument dépend d'une situation fictive assez organisée, et les actes psychiques qui en déterminent la formation sont des assomptions. Nous y procédons par raisonnements hypothétiques (qui pour Meinong sont des « inférences assomptives ») plus ou moins implicites et qui s'impliquent à partir des faits fictifs (l'existence de Harpo, de son handicap, de sa possession de l'intégralité du savoir scientifique portant sur les sons, de sa guérison et de ses nouvelles expériences), mais au sein desquels des raisons épistémiques sont articulables. Nous avons bien procédé par assomptions mais nous semblons, à partir de là, pouvoir obtenir un argument important contre le physicalisme (il y a des contenus de connaissance qui ne semblent pas être exprimables en termes d'informations physiques), argument formulé avec une certaine force et toujours discuté. Le gain épistémique des expériences de pensée semble ainsi dépendre de la capacité des assomptions à former les situations fictives complexes caractéristiques, de manière à faire se révéler des raisons de soutenir ou de rejeter des thèses.

ASSOMPTIONS ET CONNAISSANCE (III) : MODELES SCIENTIFIQUES

${ }^{10}$ Howard Robinson, "Dualism", The Stanford Encyclopedia of Philosophy (Fall 2017 Edition), Edward N. Zalta (éd.), URL = <https://plato.stanford.edu/archives/fall2017/entries/dualism/>. 
Meinong affirme aussi que les assomptions jouent un rôle en science. Il souligne que les hypothèses, à un certain stade de leur formation, sont des assomptions, mais il nous faut peut-être préférer ici le cas des modèles. Les hypothèses, à un certain degré d'élaboration, supposent un rapport au réel et ont des conditions de correction, tandis que l'assomption porte sur des entités fictives. Les modèles scientifiques correspondent mieux à de telles entités fictives. Classiquement, ils sont tenus pour des entités intermédiaires entre les phénomènes et la théorie, de manière à aider à rendre la théorie applicable ou les phénomènes interprétables. En tant que tels, ce sont des sortes d'idéalisations ou des représentations qui sont simplifiées sous certains rapports, au sens où elles encodent certains traits du réel, et en négligent d'autres - ce sont des falsifications volontaires, comme disait David Lewis. Travailler sur des modèles, cela revient donc à penser à quelque chose d'autre que le réel lui-même (il n'est pas ici directement l'objet de la pensée) et à réfléchir sur une représentation simplifiée de certains de ses aspects, ou de certaines de ses propriétés, leur «support » étant ici «idéal ». Se trouve alors pensée une entité qui se substitue au réel et qui, pour cela, dépend d'assomptions. Juger que le modèle est une fidèle réplique de tel ou tel aspect du réel est certes épistémiquement nécessaire, mais cela relève d'une démarche différente.

Si l'on considère les travaux sur les pendules idéalisés permettant de représenter l'application des forces newtoniennes, il apparait qu'ils permettent de faire abstraction de certaines propriétés du réel et d'appliquer des formules à propos de propriétés de schémas. Le pendule idéal n'est pas le pendule réel : travailler sur le premier suppose de faire usage d'assomptions. Travailler sur le rapport du premier au second relève d'un autre type d'activité. $\mathrm{Ou}$ encore, en modélisation moléculaire, une géométrisation des structures chimiques est présentée de manière graphique et traitée informatiquement: les opérations sur les modèles restent distinctes, lors de leur exercice, des opérations sur le réel, et dépendent d'assomptions. La valeur épistémique du modèle vient du fait que nous savons qu'il y a une relation d'isomorphie entre les modélisations informatiques et les structures et propriétés véritables du réel au niveau chimique, ce qui garantit la pertinence des travaux sur les modèles.

Considérons enfin l'exemple du modèle de l'atome de Niels Bohr de 1913. Lors de sa création, il entrait en contradiction avec certains aspects de la théorie physique de l'époque. Il représentait des traits hypothétiques de l'atome, sans avoir à les justifier en regard de la théorie de l'époque, vis-à-vis de laquelle il présentait des incohérences. En effet, suite au modèle de Rutherford, l'atome était supposé être constitué d'un noyau et d'un nuage d'électrons. Les lois de l'électrostatique impliquaient que des forces s'annulent et fassent se maintenir la relation entre le noyau et l'électron. Or le mouvement supposé des électrons crée théoriquement une déperdition d'énergie et modifie donc ce rapport électrostatique, ce qui devait théoriquement conduire les électrons à s'écraser sur le noyau 
et à faire s'effondrer l'atome sur lui-même. Devant cette incohérence entre théorie et données expérimentales, Bohr propose une sorte de fiction réglée qui indique des raies spectrales produites lors de trajectoires des électrons et définit des niveaux d'énergie rendant compte des positions des électrons. Ce modèle parfois nommé " prospectif » est ainsi solidaire de données expérimentales et d'hypothèses de quantification des niveaux d'énergie allant contre la théorie de l'époque, ainsi que des propriétés de l'atome qui ne sont pas traitées par la théorie. C'est une fiction très rationalisée. On suppose ici explicitement que le modèle n'est pas identique à la réalité de l'atome, mais qu'il présente un ensemble de caractéristiques étudiables sans que l'on ait à juger de l'existence de leur substrat, ni de l'affirmation, par la théorie, des relations entre les propriétés en question et ce que l'on sait du substrat existant. Sont ainsi étudiées des caractéristiques ancrées sur une sorte d'objet non-existant mais assurément pensé, et qui, pour son statut d'objet d'investigation, dépend d'assomptions: car il ne s'agissait pas ici de simplement se représenter un atome, ni de juger que l'atome était ainsi et ainsi. Il s'agissait plutôt de viser quelque chose dont on savait qu'il n'y avait rien qui existât à l'identique, et de lui attribuer des caractéristiques qui tiennent compte d'informations, et d'autres qui sont construites sur la base de raisons. En ce sens, nous avons bien affaire à des états de choses complexes visés par des assomptions ${ }^{11}$.

\section{INTERMEDE :}

\section{LA MODIFICATION DE LA TRIPARTITION BRENTANIENNE}

Ce qui a été présenté jusqu'ici va dans le sens de la reconnaissance d'un droit de cité des assomptions parmi les actes mentaux et les attitudes épistémiques. Le besoin d'un acte intermédiaire entre représentations, jugements et sentiments conduit ainsi à une modification de la classification de Brentano. Meinong introduit donc ici la classe des assomptions dans ce qui devient une quadripartition. Les classes d'états psychiques se rapportent toutes à des objets de pensée: les représentations visent des objets, les jugements et les assomptions des objectifs (états de choses abstraits), les sentiments (incluant ici émotions et désirs) des valeurs ou des devoirs. Toutefois, présenter de la sorte cette classification d'actes psychiques avec leurs objets dédiés laisse de côté le fait que ces actes sont constamment entremêlés. Un jugement a pour présupposition une ou plusieurs représentations, car on juge quelque chose à propos d'autre chose, qui doit être présenté à titre d'objet et fondé sur une représentation, et il en va de même pour les assomptions, qui portent sur des objets traités au sein d'objectifs. De même les états affectifs présentent-

${ }^{11}$ Voir aussi Nadine DE COURTENAY, «The epistemological virtues of assumptions : towards a coming of age of Boltzmann and Meinong's objections to "the prejudice in favour of the actual" ? ", Studies in History and Philosophy of Science 41 (2010), p. 41-57. 
ils des objets à l'esprit - comme des valeurs pour les émotions, et des états du monde devant ou ne devant pas exister pour le désir - en étant eux-mêmes fondés sur des jugements ou des assomptions. Même en nous tenant à ces relations sommaires, nous voyons que la position des assomptions au sein de ce cette classification n'est pas là seulement pour ajouter un acte psychique, dont l'ajout se justifie en fonction des propriétés épistémiques que nous avons indiquées. Les assomptions doivent être analysées dans leurs relations aux représentations, aux jugements et aux sentiments.

\section{ASSOMPTIONS ET REPRESENTATIONS (I) : LA CONDITION DE L'INTENTIONNALITE DES REPRESENTATIONS}

Quelles relations trouve-t-on chez Meinong entre les assomptions et les représentations? Les représentations sont pour Meinong des existants mentaux. Les représentations ont classiquement des objets dont elles se distinguent, tandis que contenu et représentation entretiennent quant à eux une relation réelle : on ne peut distinguer ces derniers que par une distinction de raison. Pour être formée, une assomption doit prendre appui sur une représentation qui en est alors la présupposition : de la même manière qu'un jugement présuppose une représentation de ce sur quoi il porte, une assomption présuppose une représentation de ce sur quoi elle porte.

Cette lecture implique toutefois que les représentations aient un objet par ellesmêmes, vers lequel elles sont dirigées, exactement comme le disait Brentano. De ce point de vue, il est donc possible d'avoir une représentation présente à l'esprit, par laquelle un objet est visé, sans que cela n'implique que je juge forcément quoi que ce soit, ou que je forme une assomption envers quoi que ce soit. Il serait alors justifié d'affirmer qu'une assomption s'autorise de cette propriété d'intentionnalité de la représentation, pour qualifier son objet, en annulant toute supposition d'existence à son propos. Cela semble aller de soi mais c'est une conception que Meinong modifie à la lumière d'une autre théorie de l'intentionnalité, ou, comme il dit, d'une théorie de «l'objectualité » (Gegenständlichkeit) qui détermine les conditions sous lesquelles une représentation vise un objet. L'ordre de l'explication est alors, pour une part, inverse : les assomptions sont ici nécessaires pour que les représentations « exercent» leur intentionnalité envers un objet de manière actuelle.

Rendre compte de cela suppose de revenir sur la théorie de l'acte psychique de Meinong : tout état mental est un acte pour lui, ce terme désignant aussi bien un épisode mental qu'une expérience consciente ou une modification de l'esprit. Les états psychiques relèvent ici d'une dimension d'activité ou de passivité. Viser un objet, quel qu'il soit, cela relève d'une activité : c'est la condition pour que la propriété d'intentionnalité des représentations ait une réalisation, comme par exemple lorsque je vise quelque chose, via la perception, en étant en prise attentionnelle avec ce quelque chose. Car en tant que telle, 
une représentation est un état d'esprit marqué par la passivité de l'esprit : une idée arrive en effet quand elle veut, comme disait Nietzsche, ou bien elle est éveillée par une autre, ou par une activité de recherche, de réflexion, ou encore par une émotion elle-même sollicitée par des attentes qui sont rompues. Comme les sentiments (sauf les désirs), et à la différence des jugements et des assomptions qui supposent une activité, les représentations sont passives et ne peuvent pas, pour Meinong, être par elles-mêmes être en prise actuelle avec un objet, même si elles ont une disposition à viser un objet par le biais de leur contenu. Voici donc un trait important des relations entre assomptions et représentations. Celles-ci ont pour Meinong une objectualité (Gegenständlichkeit) potentielle: elles possèdent un contenu qui, dispositionnellement, et donc de manière non actualisée, est en connexion avec un objet. Mais cette disposition du contenu des représentations à être dirigée vers un objet n'est actuelle que si quelque autre état psychique joue le rôle déterminant, en ajoutant à la représentation la dimension d'activité qui lui manque intrinsèquement, de façon à ce qu'elle puisse viser son corrélat objectuel et le faire se présenter à l'esprit. Jugement et assomption comportent évidemment cette dimension d'activité qui fait s'actualiser la disposition des représentations à être dirigées vers quelque objet, qui ne peut alors se présenter qu'au sein d'objectifs (les états de choses meinongiens).

Il n'y a pas donc pas de simples pensées d'objet qui seraient indépendantes de jugements ou d'assomptions. Que je rêvasse, que j'imagine, que je pense à des problèmes ou à des faits futurs, mes représentations supposent des assomptions. Si l'on ajoute à cela la perspective meinongienne de l'Aussersein, c'est-à-dire la thèse disant que penser revient à sélectionner un ou des objets parmi l'infinité des objets disponibles et la gradualité ellemême infinie de leurs propriétés, toute pensée possiblement occurrente, même la plus absurde, implique d'autant plus une assomption pour orienter la représentation concernée ${ }^{12}$.

\section{ASSOMPTIONS ET REPRESENTATIONS (II) : GOMMENT APPREHENDER UN OBJET ?}

Un autre aspect de la doctrine de Meinong rend décisive la place des assomptions comparativement aux représentations. Avant même de s'intéresser à ce qu'est une pensée vraie et donc une connaissance, Meinong traite de la question de savoir comment un objet de pensée peut, précisément, être un objet de pensée, c'est-à-dire un objet visé par l'esprit, et qui lui est présenté en retour. C'est un préalable à la question de la correction des pensées et donc à celle de la connaissance. En ce sens l'épistémologie est une partie de la

${ }^{12}$ Sur l'Aussersein, voir Venanzio RASPA, "Fictional and aesthetic object : Meinong's point of view", in A. Bottani and R. Davies (éds.), Modes of Existence. Papers in Ontology and Philosophical Logic, Frankfurt, Ontos Verlag, 2006, p. 47-80. 
théorie de l'appréhension, qui la précède et la conditionne.

Les assomptions jouent ici un rôle central, outre celui d'assurer la directionnalité des pensées, car elles sont aussi les activités psychiques déterminantes pour l'appréhension des objets. Dans un fragment ${ }^{13}$ antérieur à la deuxième édition de Über Annahmen, Meinong soutient en effet qu'il y a un rapport intrinsèque entre le fait de l'appréhension et la propriété de tous les objets de relever du « hors-l'être » (Aussersein) c'est-à-dire de n'être ni des existants ni des subsistants. Etre un objet, c'est être susceptible d'être appréhendé : Meinong défend cette position afin d'éviter ce que l'on pourrait qualifier comme l'erreur représentationnaliste, c'est-à-dire l'erreur de toute approche plaçant l'objet dans la dépendance de la représentation. Or pouvoir être représenté n'est pas ce qui fait qu'un objet est un objet - c'est plutôt selon lui le fait de pouvoir être appréhendé qui détermine cela, et cette appréhension n'est alors plus une question de représentation, mais implique les autres actes de l'esprit, et en premier lieu « l'être-dirigé-vers » (Gerichtetsein) dépendant des assomptions et de leur dimension active. Dans le traité sur les assomptions, cette approche est quelque peu affaiblie et les assomptions redeviennent ainsi le facteur psychologique qui rend possible l'appréhension en vertu de la propriété présentée plus haut: celle de pouvoir activer l'intentionnalité potentielle des représentations et de les faire viser tout objet indépendamment de la considération du statut ontologique de leur corrélat objectuel.

Tout se passe donc comme si Meinong prenait très au sérieux le problème du rapport entre ce que l'on pourrait appeler « l'écueil du représentationnalisme » et le « défi de l'intégration »: si d'un côté nous ne considérons pas les objets comme dépendants de la représentation, mais que nous les tenons pour des entités qui en sont ontologiquement indépendantes, comment nous assurer, inversement, que nous avons accès à celles-ci, alors qu'elles possèderaient une telle indépendance vis-à-vis de nous-mêmes et de nos facultés ? Sur ce point, la catégorie de l'assomption permet ici d'assurer que toute pensée peut avoir un objet, au sens où elle est, en dernière instance, le déterminant de l'activité impliquée par le Meinen (la visée) : cette activité faisant qu'un objet se trouve sélectionné (parmi l'infinité des objets hors-l'être et de leur gradualité elle-même infinie) et présenté à l'esprit. La pensée est ainsi orientée vers quelque objet qui, en vertu de cette visée et de ses composantes, s'impose à l'esprit en se présentant à lui. C'est ce qui fait qu'une appréhension (Erfassung) a lieu, laquelle ne crée pas les objets mais les sélectionne. «Visée » et « présentation » sont ainsi les deux concepts centraux de la théorie de l'appréhension.

Conclusion sur ces points: les assomptions, ainsi liées au refus de faire des

13 "Über Inhalt und Gegenstand" [Sur le contenu et l'objet] in Gesamtausgabe VIII : Kolleghefte und Fragmente. Schriften aus dem Nachlass, R. Fabian and R. Haller (éds), 1978 (Fragment de 1908). 
représentations des états d'esprits dotés par eux-mêmes d'une intentionnalité actuelle, ont donc un rôle plus fondamental pour cette intentionnalité même des représentations et la visée d'états de choses. Elles sont de plus essentielles à la théorie de l'appréhension de Meinong, laquelle tend à défaire le lien classique entre représentations et objets.

\section{DES ASSOMPTIONS DISPENSABLES (I)? MEINONG ET LA THEORIE BRENTANO-MARTYENNE DU JUGEMENT}

Les rapports entre assomptions et jugements ont une autre complexité et questionnent plus urgemment la question de la dispensabilité des premières. Cette question est largement illustrée par un désaccord théorique de Brentano et surtout de Marty, son défenseur, avec la position de Meinong. Brentano soutenait la thèse de la réductibilité de tout jugement à des jugements existentiels ${ }^{14}$, puis a précisé cette doctrine, où la thèse du double jugement, plus tardive, était selon lui implicitement présente. Pour les brentaniens, tout jugement consiste d'abord à affirmer l'existence ou la non-existence de quelque chose, et le contenu de ce jugement doit alors faire lui-même l'objet d'un nouveau jugement consistant à lui attribuer un prédicat ou à nier cette attribution. Meinong s'est d'emblée opposé à cette approche en raison de sa position sur les jugements relationnels. Il pense dès ses premiers textes qu'un jugement relationnel peut être vrai même si les éléments en relation n'existent pas. C'est par exemple le cas du jugement que l'on peut porter sur la différence entre deux couleurs qui sont simplement imaginées et qui n'ont pas d'existence effective : le jugement est vrai mais ne porte pas sur des existants. Au sein de cette distinction entre jugement existentiel et jugement relationnel, héritée de Hume, les jugements catégoriques sont tenus comme une espèce des jugements relationnels ${ }^{15}$. Ceci est à l'arrière-plan de la question de savoir si pour les brentaniens, les assomptions sont dispensables ou non: selon eux, elles sont inutiles et leur supposée spécificité ne traduit rien que le jugement ne puisse pallier. Là où Meinong pense un accès direct par

\footnotetext{
${ }^{14} \mathrm{Ce}$ point était le cœur de la nouvelle logique de Brentano, sur laquelle un programme scolaire devait se constituer. Malheureusement, Höfler (aidé par Meinong) a publié sa Logik avant cela, laquelle épousait une direction différente et soutenait l'irréductibilité des jugements catégoriques aux jugements existentiels, remettant donc en cause aux yeux des autorités la valeur de la nouvelle logique. C'est une des raisons de l'attitude constamment critique de Marty envers Meinong. Meinong a aussi donné une recension du livre de Hillebrand (Die neuen Theorien der kategorischen Schlüsse, Alfred Hölder, Wien, 1891) sur cette réductibilité des jugements catégoriques aux jugements existentiels, dans laquelle il soutient qu'il n'est pas possible d'opérer cette réduction sans omettre des éléments centraux des jugements catégoriques. Il soutenait aussi que les objets des deux types de jugements ne peuvent pas être identifiés l'un à l'autre. Sur ce dernier point, voir Meinong, 1892, "Rezension von Franz Hillebrand, Die neuen Theorien der kategorischen Schlüsse", Gesamtausgabe, Bd 7, R. Haller (ed.), Akademische Druck- und Verlagsanstalt, Graz, p.197-222.

${ }^{15} \mathrm{En}$ effet, juger que $\mathrm{X}$ est $\mathrm{A}$, cela revient à juger que $\mathrm{X}$ est en relation avec $\mathrm{A}$, ou que $\mathrm{A}$ entretient une relation d'inhérence avec $\mathrm{X}$.
} 
le jugement à un état de choses, composé d'un objet à propos duquel quelque chose est jugé - l'état de choses étant ce qui est jugé -, Marty suppose un premier acte de jugement affirmant l'existence de ce qui est jugé et un second acte qui affirme un prédicat du contenu du premier jugement.

Ce type de pensée soutenu par Marty correspond de plus à une approche dont Meinong s'est très vite désolidarisé après l'avoir trouvée chez Brentano, mais aussi chez un kantien psychologisant et logicien comme Sigwart, ou chez Stumpf, ou encore chez Husserl dans sa Philosophie de l'arithmétique. Inspiré par Kant et sa thèse de l'unité de l'aperception et de la réflexion conduisant à une unité de conscience, Sigwart ${ }^{16}$ soutenait que l'unité des pensées repose sur un processus de réflexion visant l'acte de pensée, processus qui confère l'unité aux pensées. C'était grosso modo aussi le cas de Husserl qui soutenait par exemple en 1891, dans sa Philosophie de l'arithmétique, que seule la réflexion sur l'acte psychique reliant des représentations discrètes pouvait fournir une pensée unifiée de celles-ci et donc fonder la représentation d'une pluralité en tant que pluralité ${ }^{17}$. Dès son article de 1891 sur la psychologie des relations et des complexions ${ }^{18}$, Meinong écarte ce type d'approche qui ne consiste, selon lui, que dans un détour réflexif théoriquement inutile et phénoménologiquement non attesté, voire contraire à l'expérience. Il restera très critique, dans tous ses écrits, à propos de ce genre de thèses.

Le point commun à ces pensées, que l'on retrouve dans la théorie du double jugement Brentano-martyen, est qu'elles supposent qu'un premier acte mental peut offrir un contenu qui fait alors réflexivement l'objet d'une autre opération, cette opération permettant d'assurer à la fois l'unité de la représentation et l'intentionnalité ou la visée objective de l'ensemble ${ }^{19}$. La perspective judicative de Brentano-Marty reprend le même processus : tout jugement implique une affirmation d'existence, donc un premier

\footnotetext{
${ }^{16}$ Christoph VON SigwarT, Logik, vol. 1, Die Lehre vom Urtheil, vom Begriff und vom Schluss, Tübingen, J.B. Mohr (Paul Siebeck), 1873, p. 36 : “L'idée de la difference n'est pas donnée. [...] L’idée de la différence, ou celle de l'identité, ou de la diversité, n'apparaît que lorsque la distinction est consciemment saisie et qu'il y a reflexion sur cette activité."

${ }^{17}$ Husserl, Philosophie der Arithmetik, Pfeffer, Halle-Salle, 1891 (trad. fr. J. English, Philosophie de l'arithmétique. Recherches psychologiques et logiques, Paris, Puf, 1972, voir notamment le $3^{\text {eme }}$ chapitre et le $\S 46$.

${ }^{18}$ Meinong, "Zur Psychologie der Komplexionen und Relationen", in MeINong 1968-78, Gesamtausgabe, R. Haller (ed), Akademische Druck- und Verlagsanstalt, Graz, Vol. I, p. 279-303.

${ }^{19} \mathrm{C}$ 'est d'une certaine manière assez proche de la théorie de la « représentation concomitante » de Brentano, comme appliquée au jugement. Une représentation, visant un objet, éveille une représentation de second ordre qui vise la première ainsi que la visée de celle-ci, produisent donc une représentation objectuelle et consciente dans une unité de conscience, précisément. Pour ce qui concerne les représentations, Pour Meinong, il y a ici un problème de régression à l'infini, car si un acte pour viser un objet a besoin d'un autre acte qui le prend pour objet, alors il n'y a pas de raison de penser qu'un nouvel acte n'est pas nécessaire pour assurer que le deuxième acte capture bien le contenu du premier, etc.
} 
jugement, dont le contenu est réflexivement saisi par l'esprit afin de former un deuxième jugement, prédicatif, englobant le second ${ }^{20}$. Ceci est parfaitement vain pour Meinong et revient à identifier indûment l'objet d'une affirmation d'existence et l'objet des pensées en tant qu'objet. Car dans cette optique, ne pas être jugé comme existant revient, pour un objet, à ne pas être tenu pour un objet tout court : c'est ce que Meinong refuse. A ce type d'approche il oppose de plus l'irreprésentatiblité des expériences internes, et l'inutilité théorique d'un tel processus de détour, dont aucun témoignage phénoménologique non partisan n'a jamais attesté. Cet argument se retrouve fréquemment chez Meinong comme chez Moore ${ }^{21}$, lorsqu'il est question de la visée d'un objet et d'un détour par les représentations. L'apparition des assomptions qui permettent de penser des objets n'existant pas, et à propos desquels on peut émettre des jugements sur leur possession de certaines caractéristiques, est une théorie allant complètement à l'encontre de la théorie des actes psychiques impliquant une réflexivité.

\section{DES ASSOMPTIONS DISPENSABLES (II)? L'INCOMPATIBILITE DES ACTES PSYCHIQUES SELON BRENTANO ET MARTY}

Brentano et Marty ne pouvaient donc que développer une approche critique envers les assomptions, en affirmant par exemple qu'elles impliquent des actes mentaux incompatibles au sein d'une même pensée. Marty $^{22}$ argumente sur ce point comme il suit.

\footnotetext{
${ }^{20}$ Pour les non-existants : A juge existentiellement que B existe jugeant qu'il y a un pégase et A juge réflexivement que $\mathrm{B}$ fait erreur : il n'y a pas d'objet tel que Pégase.

${ }^{21}$ George Edward MoORE, «The nature of judgement », Mind, New Series, vol. 8, n 30 (1899), p. 179 : « Ainsi, lorsque je dis " Cette rose est rouge », je n’attribue pas une partie du contenu de mon idée à la rose, et je ne suis pas plus en train d'attribuer des parties du contenu de mes idées de la rose et du rouge à un troisième sujet quelconque. Ce que j'asserte ainsi, c'est une connexion spécifique de certains concepts qui forment le concept intégral de " rose » avec les concepts de «cette », " maintenant » et " rouge », et le jugement est vrai s'il existe une telle connexion. ».

22Dans la recension peu favorable qu'il a donnée de la première édition de l'ouvrage de Meinong, Marty écrit : « Si non-A est une assomption prédicative ( = quelque chose qui n'est pas A), le sujet est par là accepté. Après tout, il est impossible de prédiquer quelque chose d'un sujet ou de nier quelque chose de celui-ci sans l'accepter. Si, toutefois, une telle assomption est impliquée comme un élément de tout jugement concernant un ainsi-dit concept négatif, c'est aussi le cas dans le jugement « il n'y a pas de non-A ». Et cela signifie que dans l'attitude psychique, qui rejette qu'il y ait quelque chose qui ne soit pas A, l'acceptation que quelque chose est et n'est pas A est contenue en tant qu'élément constitutif et ses éléments se trouvent en contradiction les uns avec les autres. (...) nous avons en fait devant nous en un exemple de position dans la négation opposée une conduite contradictoire, que les éléments ensemble soient appelés un jugement ou l'un d'entre eux un «assumer » et l'autre seulement un juger. » (Anton MARTY, "Über Annahmen", Zeitschrift für Psychologie und Philosophie der Sinnesorgane, 40, 1905, p. 32) pour les critiques de Brentano, un peu différentes dans le détail mais qui en partagent l'esprit, voir par exemple la Psychologie du point de vue empirique, Vrin, 2008, p. 296-297.
} 
Revenant sur la formule « il n'y a pas de non-A », laquelle semble affirmer la négation de quelque chose qui est supposé (assumé) être un non-existant, il l'interprète au prix d'un amalgame entre le fait de " viser un objet », " affirmer son existence » et « le reconnaitre ». La reconnaissance est un concept de la théorie du jugement (tout comme le rejet) que Marty utilise ici : reconnaître un objet, pour Marty, ne revient pas à en faire l'objet d'une l'assomption au sens meinongien. Cela conduit plutôt à interpréter non-A comme étant quelque chose, car prédiquer quelque chose d'un objet présuppose de le reconnaitre. Marty traduit donc Non-A de la sorte : « quelque chose n'est pas A », ce qui signifie chez lui « il y a quelque chose qui n'est pas A ». Chez Meinong, " quelque chose qui n'est pas A » implique que l'objet, le quelque chose, soit certes visé, mais au sens assomptif, au sens de la visée d'un objet tout court. Marty nie subrepticement cela et affirme que la formule « il n'y a pas de non-A » est équivalent à « il n'y a pas quelque chose qui n'est pas A », ce qui aboutit donc à «il n'y a pas et il y a quelque chose qui n'est pas A. ", c'est-à-dire à une pensée qui implique deux attitudes psychiques incompatibles, celle de la reconnaissance et celle du rejet, ici à propos de A. Ce qui n'est pas vraiment une situation des plus enviables lorsqu'il s'agit de rendre compte des attitudes psychiques.

Schématiquement :

- Marty identifie « viser un objet par assomption » et « affirmer son existence, c'està-dire le reconnaître »

- Prédiquer quelque chose d'un objet présuppose de le reconnaître

- (1) Non-A = quelque chose n'est pas A

- (2) Quelque chose n'est pas A = il y a quelque chose qui n'est pas A

- (3) Il n'y a pas de non-A = Il n'y a pas quelque chose qui n'est pas A = Il n'y a pas et il y a quelque chose qui n'est pas A

- Reconnaissance et rejet de A en même temps = incompatibilité de deux actes psychiques

Nous trouverions donc chez Meinong, d'après Marty, à la fois la reconnaissance et le rejet de A, c'est-à-dire une incompatibilité entre attitudes psychiques rendant intenable la position du philosophe de Graz. Afin d'éviter cette conséquence indésirable, Marty soutient qu'il suffit de rapporter à la notion de jugement ce qui, chez Meinong, passe pour une assomption - c'était implicite dans sa critique. Ainsi, ce premier jugement « quelque chose qui n'est pas A », contient déjà l'affirmation que c'est un non-A, et le deuxième jugement disant qu'il n'y a pas de non-A, donc qu'il n'y a pas quelque chose qui n'est pas A, nierait réflexivement le premier, sans faire pour autant tomber l'esprit dans des 
attitudes contradictoires ${ }^{23}$.

Il est évident que l'identification à laquelle a procédé Marty entre « quelque chose » et « il y a », où le « il y a » est entendue au sens de la reconnaissance, donc de l'affirmation d'une existence, ce qui implique alors des attitudes incompatibles. Meinong n'aurait toutefois jamais voulu dire que quelque chose qui n'est pas A implique le moindre jugement préalable envers l'existence de quelque chose, ou sa « reconnaissance » - si ce n'est comme un objet dont le statut existentiel est précisément neutralisé en tant qu'objet visé par une assomption. La spécificité psychologique de l'assomption consiste précisément à penser quelque chose sans rien supposer d'autre qu'objet visé par l'esprit, et rien qui soit « reconnu » comme existant, subsistant (c'est juste un objet visé) - ce que Bertrand Russell, dans son article "Meinong's theory of complexes and assumptions », avait fait valoir après avoir relevé la même difficulté possible que celle que présente Marty. Il remarquait que deux attitudes psychologiques incompatibles pourraient sembler être impliquées ici. Mais il avait immédiatement écarté cette possibilitée ${ }^{24}$, en tenant tout simplement compte de la théorie des assomptions elle-même et donc de la neutralité existentielle de l'objet, précisément, des assomptions, laquelle exclut la possibilité d'avoir quelque chose comme une « reconnaissance » et un « rejet » en même temps.

\section{DES ASSOMPTIONS DISPENSABLES (III)? LES ASSOMPTIONS COMME CONDITIONS DES JUGEMENTS}

Dans la deuxième édition de Über Annahmen, Meinong précise le sens de sa théorie des assomptions contre diverses critiques, et en la présentant comme une alternative puissante à la théorie des doubles jugements. Outre l'inutilité du processus réflexif, et son incompatibilité totale avec l'expérience ordinaire de la pensée, Meinong oppose une autre critique aux partisans de la primauté du jugement d'existence. Ils présupposent une attitude assomptive, qui au cœur de leur approche, fonctionne comme une condition de possibilité de leur thèse. La stratégie de Meinong consiste à soutenir que les jugements d'être, ou jugements existentiels, ou encore jugements qui dans la position Brentanomartyenne affirment un sujet, impliquent des assomptions qui jouent un rôle déterminant pour que l'objet du jugement en question soit bien donné. La thèse que l'affirmation par le jugement de l'existence de l'objet précéderait la saisie de ses caractéristiques est donc directement mise en question : comme dit Meinong, ce qui est mystérieux dans la position brentanienne, c'est qu'un objet puisse être appréhendé, même dans la perception, sans

\footnotetext{
${ }^{23}$ Sur ce débat, voir aussi Sébastien RichaRD, « Marty against Meinong on Assumptions », in Hamid Taieb \& Guillaume Fréchette (éds.), Mind and Language - On the Philosophy of Anton Marty, De Gruyter, 2017, p. 219240.

${ }^{24}$ Bertrand RusselL, « Meinong's theory of complexes and assumptions », Mind, 1904, n 51, p. 338-339.
} 
que soit considérée son appréhension via un objectif d'être-tel, autrement dit via un état de choses où est indiqué ce qu'il est d'abord.

Toute visée, y compris maintenant celle qui concerne des portions de réalité comprises sur le mode de l'effectivité et données, apparemment, au sein de la perception, relèvent en fait du même rapport entre assomption et objectif d'être-tel que celui que l'on trouve dans le cas de la considération des complexes non-existants ou des définitions. Au sein de l'effectivité, donc là où les jugements d'être sont supposés être le paradigme, on ne peut en réalité pas faire l'économie des assomptions et des objectifs qualitatifs, lorsque l'on entend rendre compte du rapport cognitif actuel aux entités données dans la perception. Celle-ci est donc solidaire d'un jugement d'existence et d'une assomption d'être-tel au moins : elle a une structure propositionnelle mais au sens où, là aussi, la saisie des objets se fait par les propriétés - Meinong est en accord avec Aristote ${ }^{25}$ sur ce point sans affirmer comme lui que cela implique de soutenir que toujours le sujet concerné soit. De ce point de vue, ne pas considérer que la saisie d'un objet passe par les propriétés qu'il possède - où que l'on suppose qu'il possède - indépendamment de son affirmation d'être, c'est se condamner à ne pas comprendre comment une visée portant uniquement sur l'être du sujet, sans considérer ses propriétés, peut bien être pertinente. ${ }^{26}$

Ces objectifs d'être-tel peuvent être implicitement présents pour Meinong mais on ne peut en faire l'économie quand on voudrait privilégier une visée simplement existentielle. L'ontologie meinongienne est donc aussi impliquée dans les objets de la perception: toute perception apparemment simple a en réalité une nature propositionnelle. L'ontologie de la complexité présente très tôt dans la pensée meinongienne est ici manifeste, et elle implique toujours quelque diversité au sein de la pensée mais aussi, donc, de la perception, et la détermination de la structure du perçu dépend des objectifs en jeu - ici les entités complexes qui y sont objectivées. De ce point de vue, à ses critiques qui soutenaient que l'affirmation d'un sujet est nécessaire à toute prédication, Meinong oppose donc le caractère "aveugle " d'une saisie d'un sujet qui serait entièrement déconnectée de celle de ses propriétés, et dont on affirmerait qu'il est avant de le caractériser après coup.

\section{ASSOMPTIONS ET SENTIMENTS (I) :}

\footnotetext{
${ }^{25}$ C'est-à-dire pour le Stagirite par les notions universelles. Cf. ARistote, Métaphysique, A, I, 981 a sqq. ${ }^{26}$ MeinONG (1910), p. 274-5 : « [...] ce qui se trouve être plutôt remarquable est qu'une saisie selon le simple être, c'est-à-dire sans emploi explicite de l'être-tel, soit possible. Nous pouvons seulement supposer que même dans les données de la simple représentation, ensemble avec la visée par être qui est fondé sur elles, des objectifs d'être-tel sont au moins impliqués. Par exemple, si je perçois la porte de ma chambre, alors sans aucun doute je fais d'abord l'expérience d'une saisie d'un objet via une visée d'être. Mais personne ne nierait que des déterminations d'être-tel comme être brun, être rectangulaire, etc., sont impliquées [...] ».
} 


\section{LE PROBLEME DE LA MOTIVATION DU DESIR}

Tout comme les représentations et les jugements, les sentiments (émotions et désirs) entretiennent chez Meinong des relations avec les assomptions. Nous nous en tiendrons ici à la théorie des sentiments qui signale une difficulté dans la détermination des relations entre émotion, désir et valeur. Le problème peut être présenté comme il suit. Il semble que l'on désire usuellement quelque chose qui est tenu pour plaisant, ou doté d'une certaine valeur. Or Meinong remarque que le plaisir en question ne peut apparemment pas être présent avant que l'objet lui-même ne soit atteint. C'est un problème, car si le plaisir est ce qui détermine le désir, au sens où il est constitutif de la motivation intrinsèque à ce dernier en l'orientant vers un objet non présent, une sorte de paradoxe se présente : je ne peux obtenir le plaisir que lorsque j'atteindrai l'objet du désir, c'est-à-dire après que la motivation a fait son office, or le plaisir est supposé me motiver et être ce qui détermine le désir. Si je ne peux pas le vivre avant d'atteindre l'objet de mon désir, qui le présuppose comme condition de l'appétit, mon désir ne peut jamais être motivé, son but jamais atteint, le plaisir jamais vécu. C'est la forme générale du problème ${ }^{27}$. Chez Meinong, il a une forme plus spécifique et il est gouverné par la difficulté que représente l'articulation correcte des catégories psychologiques dont nous disposons pour en rendre compte.

Le désir, pour Meinong, porte sur un objet non-existant, au sens où il fait rechercher l'existence de quelque chose ou son inexistence, mais aussi le maintien de quelque chose dans l'être afin qu'il ne devienne pas autre chose que ce qu'il est ${ }^{28}$. Il s'agit donc de faire advenir un état du monde possible, perçu comme devant être, contrairement à d'autres. Une donnée importante est que le désir ne crée pas la valeur chez Meinong : ce n’est pas lui

\footnotetext{
${ }^{27} \mathrm{Ce}$ problème est assez classique : il était formulé par Spinoza, qui passait par l'imagination pour lui donner une solution, car elle est capable de rendre présent ce qui ne l'est pas encore réellement, en rabattant le temps de la chose, alors imaginée et rendue présente à l'esprit, sur le temps du corps, et donc d'orienter la motivation vers l'obtention de la chose (Par exemple, dans L'éthique, Livre III, la proposition 18 avec ses implications et ramifications). William K. Clifford formulait aussi ce problème dans « On the scientific basis of morals » (Lectures and Essays, L. Stephen \& F. Pollock (éds.), Macmillan and co, 1872, vol. II, p. 109-111) : comment la représentation d'un état de choses à venir, ou des conséquences d'action à venir, pourraientelles me motiver, alors que le plaisir des conséquences ne sera vécu que lorsque ces conséquences seront présentes ? En tant que telles, les représentations des conséquences prises en elles-mêmes ne peuvent pas rendre compte de la motivation que j'ai à les viser. Sa solution passe par sa théorie du « soi », dont une des fonctions est, selon lui, de substituer au plaisir à venir un plaisir tiré de la relation du soi à ces représentations de conséquences, par où elles sont tenues pour bénéfiques pour moi. Ce plaisir se substitue au plaisir brut des conséquences, puis il est associé à celles-ci.

${ }^{28}$ Il a pour objet ce que Meinong appellera un « désidératif » dans ses œuvres suivantes, c'est-à-dire un état de choses qualifié modalement au sens où il est saisi sous un aspect déontique, qui le qualifie : désirer quelque chose c'est désirer que quelque chose qui n'est actuellement pas, devienne existant ou subsistant, et cela en vertu d'une quête qui peut être celle du devoir ou dépendre de raisons de divers ordres.
} 
qui colore axiologiquement ce sur quoi il porte. Si un état du monde futur n'est pas tenu comme doté de valeurs parce qu'il serait désiré, l'ordre de l'explication entre désir et valeur requiert donc de passer par les émotions. L'émotion en général fonctionne en effet comme un état affectif qui est un révélateur de valeurs, et chez Meinong, qui est un des théoriciens ayant popularisé cette idée, ${ }^{29}$ nous trouvons un mode émotionnel de présentation des valeurs où elles sont considérées comme des objets spécifiquement présentés par les émotions et dépendants d'autres objets. L'occurrence du sentiment peut être double : un sentiment de valeur porte sur une existence ou une inexistence, au sens où il affirme l'existence ou l'inexistence de la valeur au sein d'un état de choses. Un sentiment esthétique au contraire porte par exemple sur une des propriétés de quelque chose, indifféremment à son existence ou de son inexistence. Ainsi, avoir un sentiment de valeur à propos d'un état du monde, non réalisé, détermine-t-il le désir que cet état du monde soit réalisé, par lequel est alors formée la pensée qu'il faut qu’il soit le cas ? Ce serait une solution simple au problème de la motivation du désir par le plaisir tel que nous l'avons formulé. Meinong ne le penserait toutefois pas car la relation entre les sentiments (valeur et désir) et les actes psychiques (représentation, assomptions, jugements) doit être précisément décrite, et les caractéristiques des émotions et des désirs, en dépit des apparences, ne permettent pas de surmonter le problème.

En effet, pensant à un état du monde supposé être désirable mais qui n’existe pas, et où certaines propriétés de cet état du monde sont ce qui me le fait émotionnellement qualifier comme bon, j'ai alors affaire à un état de choses qui n'existe pas mais où une valeur est en jeu et supposée être présentée émotionnellement. C'est précisément ce qui pose problème sur le plan de l'explication. Imaginons par exemple, que je pense à un monde où les relations entre la Corée du Nord et les Etats-Unis sont complètement apaisées : quel rapport faut-il concevoir entre le fait de penser que cet état du monde serait bon, et le fait de penser que c'est un état du monde désirable? Une position intuitive consisterait à dire que penser à sa qualité moralement supérieure devrait suffire à le rendre désirable. Meinong ne nie pas que ce devrait être le cas, mais il demande toutefois comment rendre compte de cet état de fait à partir de l'économie des actes psychiques dont nous disposons. Car nous pensons ici à un état du monde qui n'existe pas, et qui devrait théoriquement n'éveiller un sentiment de valeur que lorsqu'il existera, si l'on considère strictement les propriétés des émotions qui affirment une existence ou une inexistence de valeur dans une situation actuelle. Tant que cet état n'existe pas, il ne peut pas éveiller la sorte d'émotion morale que j'ai par ailleurs devant des situations réelles,

${ }^{29}$ Notamment dans son „Über emotionale Präsentation“, in Sitzungsberichte der Akademie der Wissenschaften in Wien, Phil. hist. Klasse, 183, Abh. 2, 1917 ; réédition in Gesamtausgabe III, R. Haller (éd.), Akademische Druckund Verlagsanstalt, Graz, p. 283-476. 
puisque c'est une situation non actuelle au sein de laquelle aucun sentiment ne peut affirmer l'existence ou l'inexistence de la moindre valeur. Sous ce rapport, une émotion, qui est supposée rendre désirable un état du monde en assurant la présentation de ses propriétés morales, et donc le faire considérer comme préférable par rapport à d'autres, ne peut tout simplement pas remplir son office en ces termes. C'est donc bien le paradoxe de la motivation du désir qui se repose ici : le sentiment de valeur supposé motiver le désir ne peut pas le motiver, car ce sentiment doit affirmer une existence, laquelle affirmation peut seulement avoir lieu lorsque l'objet du désir est atteint, alors qu'elle est supposée motiver la tension du désir vers cet objet. C'est cette relation entre émotion et désir, ou par ailleurs, entre plaisir et désir, qui doit être éclairée, alors que ce dont il est question n'est pas actuel ${ }^{30}$.

\section{ASSOMPTIONS ET SENTIMENTS (II) : \\ EMOTIONS, VALEURS, DESIRS, ET UNE SOLUTION INCORRECTE}

Les assomptions et leur rapport intrinsèque au non-existant fournissent-elles une solution à ce problème de la motivation du désir ? Admettons ici qu'un monde où les tensions entre la Corée du Nord et les Etats-Unis s'affaiblissent est meilleur qu'un monde où ce n'est pas le cas. Penser à un tel monde passe par une assomption, par laquelle la pensée vise un état de choses non actuel. Nous n'avons donc pas ici un état de chose actuel à propos duquel une émotion serait éveillée et présenterait une valeur. En quoi les assomptions peuvent-elles recevoir un rôle de substitution au jugement, dans ce cas ? Selon Meinong, cette activité psychique reviendrait à me faire imaginer que si cet état du monde existait, alors il produirait en moi un sentiment axiologique supérieur. Or un fossé demeure entre cette assomption et le désir de l'avènement de l'état du monde en question. Meinong soutient en effet qu'une assomption supplémentaire est requise : celle par laquelle je suppose que si cet état du monde était favorablement reçu par moi, alors il me plairait, et il serait donc désirable. C'est ce qui motiverait à fonder le désir actuel à partir de ce jeu d'assomptions qui procède, on le voit, de manière abstraite, mais qui est correct du point de vue des caractéristiques des assomptions. Cependant, cette approche contient selon Meinong une trop grande incohérence avec l'expérience et ne peut pas fournir la solution. Le processus décrit ne correspond pas à la phénoménologie ordinaire correspondant à cette situation. La motivation du désir ne peut aucunement y apparaître. Cela signifie que la catégorie des assomptions ne peut pas nous fournir en tant que telle

\footnotetext{
${ }^{30}$ Peut-on parler d'un plaisir en représentation, d'un plaisir représenté ? Il ne semble pas que ce soit le cas. Ce qui est désiré ne peut pas me donner du plaisir, car ce qui peut être désiré n’est précisément pas encore réalisé, tandis que pour avoir une représentation du plaisir, il faut en passer par l'expérience du plaisir, expérience qui suppose donc de s'être produite pour que j'en aie une représentation.
} 
la relation entre le sentiment et le désir : il faudrait que ses propriétés puissent être articulées autrement avec celles de l'émotion.

\section{ASSOMPTIONS ET SENTIMENTS (III) : \\ L'HYPOTHESE DES « SENTIMENTS ASSOMPTIFS »}

Face à cette exigence, seule une classe de sentiments, qui seraient aux représentations et aux émotions ce que les assomptions sont aux représentations et aux jugements, serait pertinente pour assurer la relation entre émotion et désir. Cette classe existe-t-elle? Est-elle repérable ? Elle devrait être une classe de sentiments qui se manifestent à propos d'existants non-actuels, et posséder, pour cela, les propriétés qu'ont les émotions dans leur rapport avec des existants actuels. Nous savons que les assomptions partagent avec les jugements les pouvoirs d'affirmation ou de négation à propos de leur objet, mais qu'elles s'en distinguent par leur défaut de conviction à propos de l'existence ou de la non-existence : ce à quoi s'intéresse Meinong est alors une classe de sentiments qui seraient apparentés aux assomptions, autrement dit des sentiments qui présenteraient des valeurs, négatives ou positives (en faisant donc signe vers leur existence ou nonexistence au sein de situations), mais qui auraient des propriétés similaires aux assomptions en portant sur des situations non actuelles ${ }^{31}$. Cette solution permettrait d'éviter la reconstruction du fait propre de la motivation en mobilisant seulement les qualités intellectives des assomptions, présentée précédemment, ou encore la paralysie en raison de l'absence de description correcte des faits psychologiques concernés.

Meinong défend la thèse qu'il y bien a une telle classe d'attitudes affectives : il les appelle des sentiments assomptifs (Annahmegefühlen) ou des sentiments d'imagination (Phantasiegefühlen). Nous les utiliserons ici de manière interchangeable. Afin de montrer qu'il y a bien des sentiments qui ont des occurrences sans que nous affirmions l'existence de ce qui fait leur objet, Meinong prend en considération un fait empirique supposé en être l'illustration, qui consiste dans un ensemble de situations ordinaires portant sur ce que nous appellerions aujourd'hui le paradoxe de la fiction. Face à une fiction, autrement dit face à des états de fait relevant de la littérature, de fables, du cinéma, de la bande dessinée, du théâtre, des opéras, du jeu (...), comment est-il possible de ressentir des émotions très proches de celles que je ressens devant des situations réelles alors que je sais qu'il ne s'agit pas de situations réelles ? Comment puis-je, par là, évaluer des situations dans lesquelles je ne me trouve pas personnellement engagé ? Comment puis-je aussi former des désirs

\footnotetext{
${ }^{31}$ Les sentiments de valeur concernent des « objectifs d'être », où une existence ou une inexistence se trouve affirmée. Les sentiments esthétiques concernent quant à eux plutôt les « objectifs d'être-tels », ou des états de choses fictifs présentent malgré tout des propriétés esthétiques.
} 
à propos de la situation de personnages de la littérature ou d'événements fictifs? Quels sont les ressorts psychologiques et cognitifs qui sont à l'œuvre?

Ces questions, que l'on pose ordinairement pour tenter d'expliquer nos attitudes affectives devant les situations de fiction, sont pour Meinong directement apparentées à celles consistant à se demander comment articuler émotion, valeur, et motivation du désir, et à déterminer la relation entre sentiment réel et sentiment assomptif. Witasek soutenait à ce titre que ces sentiments diffèrent en vertu des actes qui en sont les présuppositions ${ }^{32}$. Les sentiments qui portent sur les situations imaginaires, par exemple, dépendraient d'assomptions, tandis que le jugement serait l'acte fondamental pour les sentiments portant sur des situations non fictives. La différence entre les deux sentiments dépendrait ainsi de la différence entre les actes qui les sous-tendent, laquelle déterminerait les caractéristiques des sentiments dans ces deux situations, ainsi que leur phénoménologie. Bien qu'elle semble assez simple, économique, et cohérente avec sa propre doctrine des assomptions, Meinong refuse pourtant cette thèse. Selon lui, une telle proposition distingue bien assomption et jugement mais présuppose qu'un sentiment de même nature survienne sur ces différents actes : ce qui implique donc que la peine ressentie pour la situation tragique d'un personnage de fiction ne présente qu'une différence de degré avec la peine que nous ressentons devant la situation tragique d'un être réel. Certaines caractéristiques intrinsèques des sentiments sont au contraire réellement distinctes pour Meinong : bien qu'ému devant la détresse du colonel Chabert dans le texte de Balzac, je ne le suis pas au même titre que devant celle d'un enfant gravement malade ou une victime d'une injustice réelle et tragique. Nous serions tentés de dire que seule l'intensité du sentiment diffère. Meinong soutient au contraire que c'est la qualité même du sentiment qui fait différer les deux sentiments. Meinong est donc en accord avec l'intuition classique selon laquelle les émotions nées de la fiction et portant sur certains de ses aspects sont bien distinctes de celles nées face au réel et portant sur certains de ses aspects. La difficulté semble alors être plus forte car ces sentiments d'imagination sont supposés présenter des situations non existantes comme dotées une valeur intrinsèque afin de motiver le désir relativement à cette situation. ${ }^{33}$

Que le sentiment de l'imagination soit de même nature que le sentiment réel aurait peut-être facilité la compréhension de la relation entre les deux, mais ce n'est apparemment pas un problème pour Meinong, qui entend maintenir l'existence d'une

32Stephan WITASEK, Grundzüge der allgemeinen Ästhetik, Barth, Leipzig, 1904.

${ }^{33}$ Meinong remarque aussi, contre Witasek, que rappeler un jugement de mémoire et une émotion construite sur un tel jugement, à propos d'un fait passé, revient à produire un sentiment d'imagination sans que celui-ci ne soit produit par une assomption. Les présuppositions sur lesquels il est construit sont celles du jugement passé, et le sentiment lui-même, actuellement vécu, est un sentiment d'imagination, car la situation n'existe plus. 
différence ici sans que que celle-ci soit rédhibitoire pour rendre compte de la motivation. En effet, la différence qualitative entre les sentiments portant sur des situations de fiction et ceux portant sur des situations réelles ne signifie pas qu'ils ne partagent pas certaines propriétés pertinentes pour notre problème. Selon lui, la relation entre émotion et désir qui est manifeste face à une situation actuelle sollicitant un sentiment réel serait ainsi l'une des caractéristiques que l'on retrouve aussi dans le cas des sentiments assomptifs : malgré leur différence de nature, ils seraient aussi caractérisés par une relation intrinsèque au désir. Les sentiments assomptifs sont alors à la fois éveillés à propos de situations non existantes et motivants pour le désir qui, par là, vise d'autres situations non existantes.

\section{ASSOMPTIONS ET SENTIMENTS (IV) : EVALUER ET APPRECIER}

Les sentiments d'imaginations ou assomptifs comportent en ce sens des caractéristiques pertinentes pour notre problème. Pour indiquer la différence de nature qu'ils entretiennent avec les sentiments réels, Meinong les associe au fait d'apprécier ou d'estimer (Werten). Les sentiments réels se rapportent quant à eux au fait d'évaluer (Werthalten). Les exemples suivants illustrent cela (ce sont ceux de Meinong). Considérons que la santé ou à la richesse soient tenues pour importantes dans notre hiérarchie de valeurs. Les qualifications éthiques solidaires de ces valeurs peuvent porter sur notre cas personnel ou sur celui d'autrui. A ce titre, deux processus pour considérer ces situations (la nôtre propre et celle d'autrui) sont possibles. Une personne ici supposée être pauvre et de santé fragile « perçoit » la non-existence de son bien, et cela directement, via une émotion pénible, en raison d'un état de choses actuel où cette personne est privée de la santé et de la richesse. Cela passe par un jugement objectif sur lequel survient une émotion, laquelle qualifie la situation par une évaluation négative. Cependant, cette personne peut aussi qualifier éthiquement la situation d'une autre personne qui, elle, est riche et en bonne santé. Ici, c'est par le truchement d'un sentiment assomptif que la personne pauvre et affaiblie peut percevoir un rapport clair entre la richesse et la santé de cette deuxième personne, ce qui fait, dit Meinong, l'objet d'une appréciation axiologique ou d'une estimation ici. Cette situation n'est pas réellement implicatrice pour la personne en mauvaise santé et pauvre, et elle fait donc l'objet d'une assomption, en vertu de laquelle naît un sentiment d'imagination ou assomptif qui présente les valeurs concernées, sans que la personne pauvre et malade ne se trouve pour autant dans une situation existante, qui serait la sienne propre, et qu'elle jugerait donc (jugement qui est connecté avec la valeur qualifiant alors la situation).

Dans le premier cas, la pensée de sa propre absence de richesse et de santé, par la personne en question, dépend d'un jugement, et relève ici du registre du Werthalten (évaluer) : ce sentiment comporte la conviction de l'existence ou de la non-existence de 
quelque chose, c'est-à-dire ici la présence ou l'absence des valeurs de santé et de richesse. Dans le deuxième cas, celui de l'assomption où la première personne se considère à la place de la personne riche et en bonne santé, conduisant à un sentiment d'imagination, nous sommes dans le registre du Werten (estimer, apprécier). Il semble donc que les sentiments assomptifs permettent de déplacer la situation d'évaluation axiologique, qui implique existence et non existence, dans la dimension de situations faisant l'objet d'une assomption, par où sont éveillés des sentiments assomptifs permettant d'apprécier ou d'estimer un état de choses non réalisé ou qui ne me touche pas en première personne dans la réalité. Ces sentiments assomptifs ont ainsi des caractéristiques telles qu'ils peuvent être considérés comme des états affectifs incluant une dimension de plaisir ou de déplaisir : la situation de l'homme riche et bien portant apparaît désirable à l'individu pauvre et malade, et inversement, la situation de l'homme pauvre et malade n'apparaît pas comme désirable, mais comme à éviter pour la personne riche et bien portante. Cela découle du type de sentiment assomptif présent et se trouve alors transposable au cas de la motivation du désir : pensant à un état de choses non-actuel et éveillant un sentiment assomptif, à son propos, je dispose alors de la relation entre sentiment et désir : ce dernier porte sur l'existence désirée de cet état de choses ou son évitement. Il y a certes une différence entre les sentiments assomptifs et les sentiments liés à des situations réelles, mais Meinong semble supposer que malgré leur différence de nature, ce sont ces traits relatifs à la relation au désir qui leur sont communs. Le sentiment réel porte sur l'existant, le sentiment assomptif ne porte pas sur de l'existant mais semble comporter une tension vers l'existant et l'inexistant selon les cas en vertu de la dimension plaisante ou déplaisante qui en fait partie. En quel sens ? Le propre du sentiment assomptif est de motiver (ou de démotiver) en rendant désirable (ou repoussant) le sentiment réel qui serait vécu si l'état de choses était réalisé : imaginer, étant pauvre et malade, une situation où je suis riche et bien portant, cela rend désirable cet état de choses. C'est une propriété des sentiments assomptifs que de rendre désirables ou repoussant les affects dépendants de ces états de choses visés. Le sentiment fondé sur l'état de choses réel affirme à son propos l'existence ou la non-existence de propriétés morales, le sentiment assomptif en fait désirer ou repousser l'existence et donc avec cela le sentiment réel qui serait présent si l'état de choses avait une existence. C'est en cela que consisterait la fonction des sentiments assomptifs relativement à la dimension conative ou motivationnelle inhérente au désir.

Quoi qu'il en soit, le pouvoir motivationnel de ces « quasi-sentiments » n'est pas la moindre des choses : il fait de la connexion entre désir et valeur un fait évident, dit Meinong. Les sentiments assomptifs permettent d'assurer une continuité émotionnelle entre le sentiment de valeur, fut-il assomptif, et le désir.

\section{CONCLUSiOn}


La notion d'assomption est donc une notion que Meinong juge indispensable à toute théorie des attitudes psychiques et propositionnelles, et cela sur plusieurs plans. Cette notion permet de donner une assise psychologique à la pensée qui considère des notions, ou à celle qui procède par expériences de pensée, ou au travail scientifique sur des modèles. Sur ces points, la thèse de Meinong consiste à soutenir que les actes mentaux que sont la représentation et le jugement ne suffisent pas à rendre compte des activités psychiques en jeu dans ces situations. La notion d'assomption, par là, doit donc être ajoutée à la classification classique comportant déjà les représentations, les sentiments et les jugements. Cet ajout permet de déterminer des relations entre ces activités qui ne pourraient pas être aperçues autrement et donc de révéler des propriétés des représentations et des jugements qui, loin d'être intrinsèques, sont inséparables de leur connexion avec les assomptions. En ce sens les assomptions sont indispensables. Elles apparaissent aussi comme les vecteurs d'une compréhension et description sans cela inadéquates des relations entre désirs et valeurs, au prix d'une modification conceptuelle et d'un ajout : celui des sentiments assomptifs à la description de l'esprit. 